\title{
Correlation Analysis of Socio-Personal Profile of Dairy Farmers with Knowledge and Adoption of Improved Animal Husbandry Practices in Doda District of Jammu and Kashmir, India
}

\author{
Sheikh Umair Minhaj ${ }^{1}$, S.A. Khandi ${ }^{1}$, Rayees Ahmed Bafanda ${ }^{1 *}$, \\ Bharat Bhushan ${ }^{2}$ and Farzana Choudhary ${ }^{1}$
}

\author{
${ }^{1}$ Division of Veterinary and Animal Husbandry Extension Education, F.V.Sc\&A.H. SKUAST-J, \\ R.S.PURA, JAMMU-181102, India \\ ${ }^{2}$ Faculty of Agriculture, SKUAST-J, Chatha, Jammu-180009, India \\ *Corresponding author
}

\section{A B S T R A C T}

The present study was conducted in Doda district of Jammu and Kashmir with the objectives to study the correlation analysis of socio-personal profile of dairy farmers with knowledge and adoption of improved animal husbandry practices in Doda district. Four blocks were randomly selected using simple lottery method. The selected blocks were

\section{Keywords}

Socio-personal profile, Dairy

farmers,

Knowledge, Adoption, Improved animal husbandry practices.

\section{Article Info}

Accepted:

19 October 2017

Available Online:

10 December 2017
Kastigarh, Bhagwah, Marmat and Ghat. After preparing a comprehensive list of villages of the selected blocks, two villages were selected randomly from each of the four selected blocks. Thereafter, fifteen respondents were selected randomly from each village. Thus a total 120 respondents were selected in all as the sample. Data was collected through well structured, personal interview from the respondents either at their farm or home after proper testing of schedule and using appropriate scales. Data was then tabulated, analyzed and inferences were drawn in the light of set objectives after application of proper statistics, using various computer softwares. The study revealed young respondents have significantly higher knowledge $(55.98 \%)$ and adoption $(60.48 \%)$ than the respondents in old age. Significant negative association was observed between age and knowledge level, with correlation coefficient being -0.217 . The statistical analysis of data revealed respondents with higher education status, more diversified occupational activity, extension contact and economical motivation have significantly higher knowledge and adoption level regarding improved scientific animal husbandry practices than the respondents scoring the low in same variables. Herd size does not have significant effect on their knowledge and adoption level of respondents regarding improved scientific animal husbandry practices. Social participation mass media exposure and risk orientation of the respondents varied with the varying levels of knowledge and adoption.

\section{Introduction}

India possesses great potential in animal husbandry sector being the largest milk producing country in the world with a production of about 97.1 million tonnes (14.9\% of world, CSO, 2006). Our country also has the largest buffalo population, while we stand second in cattle population after Brazil. The per capita availability of milk has increased from $112 \mathrm{gm}$ in 1970 to $241 \mathrm{gm}$ in 2005-06 (CSO, 2006). On the other hand, 
sustained economic growth, increasing urbanization and a shift in diets are fuelling the growth in demand for livestock products. The livestock products' demand is more income elastic, with expenditure elasticity for milk being 1.47 in rural and 1.01 in urban areas (Bhalla and Hazzel, 1998). It has been estimated that by the year 2020 the demand of milk will rise to $131-158$ million tonnes (Paroda and Kumar, 2000). This would require an incremental addition of about 5 million tonnes of milk per year over next 15 years as compared to 2.5 million tonnes increment in the last 15 years (National Dairy Plan, 2021). This increased demand will have to be largely met with by increasing the productivity of the milch animals as the option of increasing their number is undesirable. Moreover, in the current scenario, livestock markets are one of the few growing markets in the world that the poor can exploit, since the demand for livestock products is estimated to double by the year 2020 (Delgado et al., 1999).

Further, the distribution of livestock in India is more egalitarian than that of land holding leading to a relatively more equitable distribution of gains from the livestock sector. In terms of numbers, marginal and small land owners ( $<2.0$ hac.) comprise $63 \%$ of rural households but account for only $34 \%$ of arable land in contrast to $67 \%$ of bovines possessed by them (Rao and Birthal,2002), a suitable condition for the equitable growth. The Gini coefficient for the income from livestock sector in India estimated to be 0.16 in comparison to 0.65 from crop sector, being closer to the value of 0.3 , which represents a relatively equal distribution of income by world standards (Sharma and Poleman, 1993). Livestock in its households not only serves the purpose of augmenting income, employment and food security but also acts as a store keeper of capital and insurance against crop failure. Further, since women constitute
$71 \%$ of the labour force in livestock farming, with 75 million women engaged in dairying against 15 million men, the development of this sector is relevant for promotion of gender equity (Desai and Mishra, 2006). Considering the need of knowledge and adoption of improved animal husbandry practices the present investigation was undertaken to assess correlation between personal and sociopersonal characteristics with the knowledge and adoption of improved animal husbandry practices:

\section{Materials and Methods}

The present study was conducted in Doda district of Jammu and Kashmir state. Doda district consists of seventeen blocks. Four blocks were randomly selected using simple lottery method. The selected blocks were Kastigarh, Bhagwah, Marmat and Ghat. After preparing a comprehensive list of villages of the selected blocks, two villages were selected randomly from each of the four selected blocks. Thus a total of eight villages were selected in all. Thereafter, fifteen respondents were selected randomly from each village. Thus a total 120 respondents were selected in all as the sample.

Data was collected through well-structured interview schedule, personal interview from the respondents either at their farm or home after proper testing of schedule and using appropriate scales. The interview schedule was developed using the package of practices of neighboring universities as "universe of content" after proper consultation with the members of Faculty of Veterinary Science and Animal Husbandry, SKUAST-Jammu.

The final schedule was divided into four broad areas namely management, feeding, breeding and health care for evaluation of knowledge and adoption in their respective sections. The management component was 
further divided into general management and clean milk production practices Data were coded, classified, tabulated and analyzed using the software; Statistical Package for the Social Science (SPSS 16.0). The presentation of data was done to give pertinent, valid and reliable answer to the specific objectives. Frequencies, percentage, mean, standard deviation, mean percent score (MPS) and Pearson product moment correlation coefficient (r) were worked out for meaningful interpretation. Mean percent score (MPS) and Pearson product moment correlation coefficient (r) were calculated by using formula:

Mean Percent Score (MPS) $=$ Observed score $\div$ Total score $\times 100$

\section{Pearson product moment correlation coefficient}

Pearson product moment correlation coefficient was computed by the following formula.

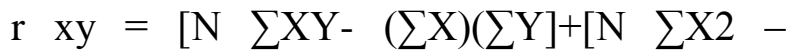
$\left.\left(\sum \mathrm{X}\right) 2\right]\left[\mathrm{N} \sum \mathrm{Y} 2-\left(\sum \mathrm{Y}\right) 2\right]$

Where, $\mathrm{X}$ and $\mathrm{Y}=$ original scores in variables $\mathrm{X}$ and $\mathrm{Y}$

Where,

$\mathrm{N}=$ number of paired scores

$\sum X Y=$ each $\mathrm{X}$ multiplied by its corresponding $\mathrm{Y}$, then summed

$\sum X=$ sum of $\mathrm{X}$ scores

$\left(\sum X\right)^{2}=$ each $\mathrm{X}$ squared and then summed

$\sum \mathrm{Y}=$ sum of $\mathrm{Y}$ scores

$\left(\sum \mathrm{Y}\right)^{2}=$ each $\mathrm{Y}$ squared and then summed

\section{Results and Discussion}

The correlation estimates between the dependent variables (i.e. knowledge and adoption) and independent variables (i.e. socio-personal profile of respondents) were presented in table 1. All the independent variables except age, though varying in extent, were positively associated with the dependent variables. Age was negatively and significantly related to the knowledge level whereas it was insignificantly related to the adoption level. Education, land holding, occupation, extension contact and economical motivation were significantly positively associated with the knowledge and adoption level of respondents. On the other hand herd size was positively but insignificantly associated with the knowledge and adoption level. Social participation, mass media exposure and risk orientation of the respondents varied with the varying levels of knowledge and adoption.

\section{Age}

For appropriate analysis the respondents were classified into three categories based on their age (i.e. young, middle and old) on the basis of means and standard deviation. Knowledge and adoption scores of respondents classified on the basis of age are depicted in the table 2. Significant negative association was observed between age and knowledge level, with correlation coefficient being -0.217 (table 1). However, age was having insignificant bearing on the adoption level of respondents. As is evident from the table 2 the majority of respondents $(55 \%)$ were middle aged with mean value of 50 years. They were followed by young (25\%) and old age group (20\%) with average age of 38 and 61 years respectively. The respondents of young age group obtained higher scores in knowledge compared to other two categories. However, the variation in adoption scores of different 
categories of respondents was insignificant. Similar findings were observed by Sayeedi (1983) for adoption and Kapse (1976) for knowledge. On contrary, Mahipal (1983) reported that the age was having positive but insignificant relationship with the knowledge and overall adoption of dairy innovations by medium farmers. The observed pattern can probably be attributed to the fact the older group seek less knowledge but adopt as per the majority. Anyhow, it is suggested that emphasis should be given to old age group to increase their knowledge and adoption, as such members play a dominant role in decision making regarding dairy sector.

\section{Education}

It is a well-known fact that education results in all-round development of an individual. Respondents were classified into three categories based on their formal education level i.e. low, medium and high (Table 3). Education was having positive and highly significant association with the improved animal husbandry practices with correlation coefficients 0.612 and 0.553 for knowledge and adoption respectively (Table 1 ).

As is evident from the table 3 the majority of respondents $(45 \%)$ were educated up to middle level, followed by high category (28\%) consisting mostly of educated above high level followed by low category (19\%). Respondents with higher formal education (above high) obtained significantly higher scores in both knowledge and adoption compared to the other two categories. Similar results were reported by other workers Hamdani (2008), Fita et al., (2012), Jeelani et al., (2015) Mikkilineni (1976), Prasad and Ponraj (2016), Sawarkar et al., (2001), and Sharma and Singh (2014). This can probably be hypothesized here that the respondents with higher formal education have increased capabilities of deciphering the complex animal husbandry information. Further the respondents with higher education status scored higher in mass media exposure, social participation etc. which could have played a role in higher scores observed.

\section{Land holding}

Land holding was having positive and highly significant association with the knowledge and adoption level (Table 1). As is evident from the table 4 the majority of respondents (98\%) were having medium land holding with 1 to 10 acres of land, followed by high land holding group (2\%) having more than 10 acres of land and low land holding group (8\%). Surprisingly respondents with medium land holding obtained higher scores of adoption and respondents with high land holding obtained higher scores in knowledge. The results are in agreement with the findings of number of workers like Hamdani (2008), Rathod et al., (2014), Sayeedi (1983), and Sharma and Singh (2014). Thus, it can be hypothesized here that resourcefulness of respondents exerts influence on the knowledge and adoption of animal husbandry practices. Also, it can be hypothesized here that the interactive mixed crop livestock farming is on the incline.

\section{Occupation}

Table1 shows that occupation was having positive and highly significant association with knowledge and adoption level. As is evident from the table 5, majority of respondents $(38 \%)$ were engaged in animal husbandry, agriculture and business occupation, followed by high category (10\%). Respondents with high occupation scores obtained significantly higher scores in both knowledge and adoption compared to other two categories. 
Table.1 Correlation estimate for dependent and independent variables

\begin{tabular}{|c|c|c|c|c|c|c|}
\hline $\begin{array}{l}\text { Independent } \\
\text { Variable }\end{array}$ & $\begin{array}{l}\text { Dependent } \\
\text { Variable }\end{array}$ & $\begin{array}{l}\text { Management } \\
\text { Practices }\end{array}$ & $\begin{array}{l}\text { Feeding } \\
\text { Practices }\end{array}$ & $\begin{array}{l}\text { Breeding } \\
\text { Practices }\end{array}$ & $\begin{array}{l}\text { Health Care } \\
\text { Practices }\end{array}$ & $\begin{array}{l}\text { Total of all } \\
\text { Dairy Practices }\end{array}$ \\
\hline \multirow[t]{2}{*}{ Age } & Knowledge & $-0.201^{*}$ & $-0.291 * *$ & -0.157 & $-0.202^{*}$ & $-0.217 *$ \\
\hline & Adoption & -0.008 & -0.093 & -0.047 & $-0.202 *$ & -0.098 \\
\hline \multirow[t]{2}{*}{ Education } & Knowledge & $0.462 * *$ & $0.533 * *$ & $0.685 * *$ & $0.477 * *$ & $0.612 * *$ \\
\hline & Adoption & $0.498 * *$ & $0.473 * *$ & $0.563 * *$ & $0.525 * *$ & $0.553 * *$ \\
\hline \multirow[t]{2}{*}{ Land holding } & Knowledge & $0.271 * *$ & $0.352 * *$ & $0.333 * *$ & $0.255 * *$ & $0.345 * *$ \\
\hline & Adoption & $0.280 * *$ & $0.210 *$ & 0.109 & 0.129 & 0.231* \\
\hline Social & Knowledge & 0.038 & 0.069 & $0.262 * *$ & $0.187 *$ & 0.152 \\
\hline Participation & Adoption & $0.222 *$ & $0.260 * *$ & $0.355^{* *}$ & $0.235 * *$ & $0.279 * *$ \\
\hline \multirow[t]{2}{*}{ Occupation } & Knowledge & $0.504 * *$ & $0.602 * *$ & $0.518 * *$ & $0.464 * *$ & $0.595 * *$ \\
\hline & Adoption & $0.572 * *$ & $0.533 * *$ & $0.457 * *$ & $0.358 * *$ & $0.560 * *$ \\
\hline \multirow[t]{2}{*}{ Herd Size } & Knowledge & 0.068 & -0.045 & -0.008 & 0.132 & 0.036 \\
\hline & Adoption & 0.003 & -0.021 & -0.042 & -0.098 & -0.029 \\
\hline \multirow{2}{*}{$\begin{array}{l}\text { Extension } \\
\text { Contact }\end{array}$} & Knowledge & $0.442 * *$ & $0.433 * *$ & $0.424 * *$ & $0.300 * *$ & $0.459 * *$ \\
\hline & Adoption & $0.313 * *$ & $0.307 * *$ & $-0.490 * *$ & $0.437 * *$ & $0.391 * *$ \\
\hline \multirow{2}{*}{$\begin{array}{l}\text { Mass Media } \\
\text { Exposure }\end{array}$} & Knowledge & 0.177 & 0.137 & 0.169 & $0.205^{*}$ & 0.193* \\
\hline & Adoption & 0.154 & 0.105 & 0.177 & $0.228 *$ & 0.169 \\
\hline \multirow{2}{*}{$\begin{array}{l}\text { Economic } \\
\text { Motivation }\end{array}$} & Knowledge & $0.693 * *$ & $0.551 * *$ & $0.512 * *$ & $0.478 * *$ & $0.639 * *$ \\
\hline & Adoption & $0.442 * *$ & $0.355^{* *}$ & $0.540 * *$ & $0.501 * *$ & $0.482 * *$ \\
\hline \multirow{2}{*}{$\begin{array}{l}\text { Risk } \\
\text { orientation }\end{array}$} & Knowledge & $0.521 * *$ & $0.435 * *$ & $0.340 * *$ & $0.330 * *$ & $0.467 * *$ \\
\hline & Adoption & 0.279 & 0.155 & 0.333 & 0.387 & 0.293 \\
\hline
\end{tabular}

* Significant at 5\% level of significance

$* *$ Significant at $1 \%$ level of significance 
Table.2 Classification of respondents on the basis of age

\begin{tabular}{|c|c|c|c|c|c|c|c|c|c|c|c|}
\hline Respondents & $\begin{array}{l}\text { Average } \\
\text { Score }\end{array}$ & $\begin{array}{l}\text { Percentage of } \\
\text { Respondents }\end{array}$ & $\begin{array}{l}\text { Dependent } \\
\text { Variable }\end{array}$ & \begin{tabular}{|l|} 
General \\
Management \\
(Mean Score \\
\pm SE) \\
\end{tabular} & $\begin{array}{l}\text { Clean Milk } \\
\text { Production }\end{array}$ & $\begin{array}{l}\text { Total } \\
\text { Management }\end{array}$ & \begin{tabular}{|l} 
Feeding \\
Practices
\end{tabular} & $\begin{array}{l}\text { Breeding } \\
\text { Practices }\end{array}$ & $\begin{array}{l}\text { Health- Care } \\
\text { Practices }\end{array}$ & $\begin{array}{l}\text { Total score of } \\
\text { all Practices }\end{array}$ & $\begin{array}{l}\text { Scores } \\
\text { obtained in } \\
\text { Percentage }\end{array}$ \\
\hline \multirow{2}{*}{$\begin{array}{l}\text { Young } \\
(30-42)\end{array}$} & \multirow[t]{2}{*}{37.8} & \multirow[t]{2}{*}{25} & Knowledge & $10.68 \pm 0.50$ & $11.80 \pm 0.61$ & $22.48 \pm 0.93$ & $24.72 \pm 1.06$ & $35.24 \pm 1.32$ & $24.24 \pm 0.68$ & $106.68 \pm 3.47$ & 58.6 \\
\hline & & & Adoption & $15.48 \pm 0.56$ & $13.80 \pm 0.49$ & $29.28 \pm 0.91$ & $16.92 \pm 0.66$ & $10.72 \pm 0.63$ & $10.12 \pm 0.42$ & $67.04 \pm 2.16$ & 57.8 \\
\hline \multirow{2}{*}{$\begin{array}{l}\text { Middle } \\
(43-55)\end{array}$} & \multirow[t]{2}{*}{49.6} & \multirow[t]{2}{*}{55} & Knowledge & $11.29 \pm 0.47$ & $11.20 \pm 0.45$ & $22.38 \pm 0.81$ & $21.53 \pm 0.80$ & $32.11 \pm 0.89$ & $23.51 \pm 0.62$ & $100.42 \pm 2.93$ & 55.2 \\
\hline & & & Adoption & $16.70 \pm 0.48$ & $13.29 \pm 0.33$ & $29.84 \pm 0.72$ & $16.80 \pm 0.61$ & $10.45 \pm 0.44$ & $9.54 \pm 0.32$ & $66.58 \pm 1.89$ & 57.4 \\
\hline \multirow{2}{*}{$\begin{array}{l}\text { Old } \\
(56-66)\end{array}$} & \multirow[t]{2}{*}{61.0} & \multirow[t]{2}{*}{20} & Knowledge & $9.65 \pm 0.83$ & $10.05 \pm 0.60$ & $19.70 \pm 1.28$ & $20.70 \pm 1.03$ & $32.95 \pm 1.29$ & $21.60 \pm 0.82$ & $96.35 \pm 3.64$ & 53.0 \\
\hline & & & Adoption & $15.80 \pm 0.83$ & $12.85 \pm 0.50$ & $29.65 \pm 1.65$ & $15.95 \pm 0.92$ & $10.90 \pm 0.63$ & $8.60 \pm 0.51$ & $64.70 \pm 3.04$ & 55.8 \\
\hline
\end{tabular}

Table.3 Classification of respondents on the basis of education

\begin{tabular}{|c|c|c|c|c|c|c|c|c|c|c|}
\hline Category & $\begin{array}{l}\text { Percentage of } \\
\text { Respondents }\end{array}$ & $\begin{array}{l}\text { Dependent } \\
\text { Variable }\end{array}$ & $\begin{array}{l}\text { General } \\
\text { Management } \\
\text { (Mean Score } \pm \\
\text { SE) }\end{array}$ & $\begin{array}{l}\text { Clean Milk } \\
\text { Production }\end{array}$ & $\begin{array}{l}\text { Total } \\
\text { Management }\end{array}$ & $\begin{array}{l}\text { Feeding } \\
\text { Practices }\end{array}$ & $\begin{array}{l}\text { Breeding } \\
\text { Practices }\end{array}$ & $\begin{array}{l}\text { Health- Care } \\
\text { Practices }\end{array}$ & $\begin{array}{l}\text { Total score of } \\
\text { all Practices }\end{array}$ & $\begin{array}{l}\text { Scores } \\
\text { obtained in } \\
\text { Percentage. }\end{array}$ \\
\hline \multirow{2}{*}{$\begin{array}{l}\text { Low } \\
(\leq 0)\end{array}$} & \multirow[t]{2}{*}{27} & Knowledge & $8.22 \pm 0.32$ & $9.56 \pm 0.46$ & $17.78 \pm 0.74$ & $16.22 \pm 0.77$ & $25.06 \pm 0.80$ & $21 \pm 0.68$ & $80.06 \pm 2.44$ & 43.98 \\
\hline & & Adoption & $12.41 \pm 0.48$ & $11.41 \pm 0.38$ & $23.81 \pm 0.78$ & $12.63 \pm 0.62$ & $9.25 \pm 0.32$ & $9.44 \pm 0.32$ & $55.13 \pm 1.90$ & 47.52 \\
\hline \multirow{2}{*}{$\begin{array}{l}\text { Medium } \\
(1-4)\end{array}$} & \multirow[t]{2}{*}{45} & Knowledge & $9.31 \pm 0.27$ & $10.37 \pm 0.42$ & $19.68 \pm 0.67$ & $19.31 \pm 0.61$ & $29.52 \pm 0.41$ & $21.42 \pm 0.49$ & $89.94 \pm 1.80$ & 49.41 \\
\hline & & Adoption & $12.26 \pm 0.41$ & $12.26 \pm 0.30$ & $24.52 \pm 0.65$ & $13.68 \pm 0.54$ & $10.57 \pm 0.21$ & $10.70 \pm 0.25$ & $59.48 \pm 1.36$ & 51.27 \\
\hline \multirow{2}{*}{$\begin{array}{l}\text { High } \\
(\geq 5)\end{array}$} & \multirow[t]{2}{*}{28} & Knowledge & $12.06 \pm 0.35$ & $14.17 \pm 0.50$ & $26.23 \pm 0.81$ & $25.12 \pm 0.68$ & $35.82 \pm 0.55$ & $27.29 \pm 0.39$ & $114.47 \pm 1.87$ & 62.89 \\
\hline & & Adoption & $17.38 \pm 0.62$ & $16.38 \pm 0.29$ & $33.76 \pm 0.84$ & $20 \pm 0.54$ & $13.35 \pm 0.29$ & $13.47 \pm 0.39$ & $80.59 \pm 1.67$ & 69.47 \\
\hline
\end{tabular}

Table.4 Classification of respondents on the basis of land holding

\begin{tabular}{|c|c|c|c|c|c|c|c|c|c|c|}
\hline Category & $\begin{array}{l}\text { Percentage } \\
\text { of } \\
\text { Respondents }\end{array}$ & $\begin{array}{l}\text { Dependent } \\
\text { Variable }\end{array}$ & $\begin{array}{l}\text { General } \\
\text { Management } \\
\text { (Mean Score } \\
\pm \text { SE) }\end{array}$ & $\begin{array}{l}\text { Clean Milk } \\
\text { Production }\end{array}$ & $\begin{array}{l}\text { Total } \\
\text { Management }\end{array}$ & \begin{tabular}{|l} 
Feeding \\
Practices
\end{tabular} & $\begin{array}{l}\text { Breeding } \\
\text { Practices }\end{array}$ & $\begin{array}{l}\text { Health- } \\
\text { Care } \\
\text { Practices }\end{array}$ & $\begin{array}{l}\text { Total score } \\
\text { of all } \\
\text { Practices }\end{array}$ & $\begin{array}{l}\text { Scores } \\
\text { obtained } \\
\text { in } \\
\text { Percentage }\end{array}$ \\
\hline \multirow{2}{*}{$\begin{array}{l}\text { Medium. } \\
(1-3)\end{array}$} & \multirow[t]{2}{*}{98} & & $9.78 \pm 0.23$ & & & & 0.49 & 0.40 & & 51.67 \\
\hline & & Adoption & $13.80 \pm 0.35$ & $13.19 \pm 0.27$ & $26.98 \pm 0.59$ & $15.21 \pm 0.44$ & $11.04 \pm 0.21$ & $11.17 \pm 0.23$ & $64.41 \pm 1.34$ & 55.52 \\
\hline \multirow[t]{2}{*}{$\operatorname{High}(\geq 4)$} & \multirow[t]{2}{*}{2} & Knowledge & $11.00 \pm 0.00$ & $11.00 \pm 0.00$ & $22.00 \pm 0.00$ & $22.00 \pm 0.00$ & $37.00 \pm 0.00$ & $26.00 \pm 0.00$ & \begin{tabular}{|l|}
$107.00 \pm 0.00$ \\
\end{tabular} & 58.79 \\
\hline & & Adoption & $11.00 \pm 4.00$ & $14.00 \pm 0.00$ & $25.00 \pm 4.00$ & $14.00 \pm 0.00$ & $9.00 \pm 0.00$ & $10.00 \pm 0.00$ & $58 \pm 4.00$ & 50 \\
\hline
\end{tabular}


Table.5 Classification of respondents on the basis of occupation

\begin{tabular}{|c|c|c|c|c|c|c|c|c|c|c|}
\hline Category & $\begin{array}{l}\text { Percent of } \\
\text { Respondents }\end{array}$ & $\begin{array}{l}\text { Dependent } \\
\text { Variable }\end{array}$ & $\begin{array}{l}\text { General } \\
\text { Management } \\
\text { (Mean Score } \\
\pm \text { SE) }\end{array}$ & $\begin{array}{l}\text { Clean Milk } \\
\text { Production }\end{array}$ & $\begin{array}{l}\text { Total } \\
\text { Management }\end{array}$ & $\begin{array}{l}\text { Feeding } \\
\text { Practices }\end{array}$ & $\begin{array}{l}\text { Breeding } \\
\text { Practices }\end{array}$ & $\begin{array}{l}\text { Health- Care } \\
\text { Practices }\end{array}$ & $\begin{array}{l}\text { Total score of } \\
\text { all Practices }\end{array}$ & $\begin{array}{l}\text { Scores } \\
\text { obtained in } \\
\text { Percentage }\end{array}$ \\
\hline \multirow[t]{2}{*}{ Low $(\leq 1)$} & \multirow[t]{2}{*}{2} & Knowledge & $14.00 \pm 0.00$ & $15.00 \pm 0.00$ & $29.00 \pm 0.00$ & $24.00 \pm 0.00$ & $35.00 \pm 0.00$ & $28.00 \pm 0.00$ & $116.00 \pm 0.00$ & 63.73 \\
\hline & & Adoption & $17.00 \pm 0.00$ & $16.00 \pm 0.00$ & $33.00 \pm 0.00$ & $21.00 \pm 0.00$ & $15.00 \pm 0.00$ & $14.00 \pm 0.00$ & $83.00 \pm 0.00$ & 71.55 \\
\hline \multirow{2}{*}{$\begin{array}{l}\text { Medium. } \\
(2-3)\end{array}$} & \multirow[t]{2}{*}{88} & Knowledge & $12.00 \pm 0.92$ & $16.00 \pm 0.89$ & $28.00 \pm 1.75$ & $28.00 \pm 1.41$ & $35.00 \pm 1.51$ & $27.00 \pm 1.28$ & $118.00 \pm 4.50$ & 64.83 \\
\hline & & Adoption & $18.42 \pm 1.05$ & $17.00 \pm 0.17$ & $35.42 \pm 1.12$ & $21.33 \pm 0.22$ & $13.33 \pm 0.22$ & $12.67 \pm 1.19$ & $82.75 \pm 1.77$ & 71.83 \\
\hline \multirow{2}{*}{$\begin{array}{l}\text { High. } \\
(\geq 4)\end{array}$} & \multirow[t]{2}{*}{12} & Knowledge & $9.48 \pm 0.21$ & $10.65 \pm 0.30$ & $20.13 \pm 0.50$ & $19.22 \pm 0.47$ & $29.52 \pm 0.50$ & $22.45 \pm 0.40$ & $91.32 \pm 1.62$ & 50.17 \\
\hline & & Adoption & $13.17 \pm 0.35$ & $12.73 \pm 0.27$ & $25.90 \pm 0.57$ & $14.40 \pm 0.43$ & $10.68 \pm 0.21$ & $10.94 \pm 00.21$ & $61.93 \pm 1.32$ & 53.38 \\
\hline
\end{tabular}

Table.6 Classification of respondents on the basis of herd size

\begin{tabular}{|c|c|c|c|c|c|c|c|c|c|c|}
\hline Category & $\begin{array}{l}\text { Percent of } \\
\text { Respondents }\end{array}$ & $\begin{array}{l}\text { Dependent } \\
\text { Variable }\end{array}$ & $\begin{array}{l}\text { General } \\
\text { Management } \\
\text { (Mean Score } \\
\pm \text { SE) }\end{array}$ & $\begin{array}{l}\text { Clean Milk } \\
\text { Production }\end{array}$ & $\begin{array}{l}\text { Total } \\
\text { Management }\end{array}$ & $\begin{array}{l}\text { Feeding } \\
\text { Practices }\end{array}$ & $\begin{array}{l}\text { Breeding } \\
\text { Practices }\end{array}$ & $\begin{array}{l}\text { Health- } \\
\text { Care } \\
\text { Practices }\end{array}$ & $\begin{array}{l}\text { Total score } \\
\text { of all } \\
\text { Practices }\end{array}$ & $\begin{array}{l}\text { Scores } \\
\text { obtained in } \\
\text { Percentage }\end{array}$ \\
\hline \multirow{2}{*}{ Low $(\leq 1)$} & \multirow{2}{*}{13} & Knowledge & $9.37 \pm 0.34$ & $11.00 \pm 0.87$ & $20.37 \pm 1.15$ & $20.75 \pm 1.05$ & $30.12 \pm 1.25$ & $21.87 \pm 1.04$ & $93.12 \pm 2.92$ & 51.16 \\
\hline & & Adoption & $14.12 \pm 0.77$ & $13.50 \pm 0.64$ & $27.62 \pm 1.36$ & $16.87 \pm 0.98$ & $11.25 \pm 0.48$ & $11.75 \pm 0.44$ & $67.50 \pm 2.98$ & 58.18 \\
\hline \multirow{2}{*}{$\begin{array}{l}\text { Medium } \\
(2-3)\end{array}$} & \multirow[t]{2}{*}{85} & Knowledge & $9.91 \pm 0.26$ & $11.33 \pm 0.35$ & $21.24 \pm 0.60$ & $20.21 \pm 0.56$ & $30.39 \pm 0.54$ & $23.14 \pm 0.45$ & $94.98 \pm 1.95$ & 52.18 \\
\hline & & Adoption & $13.74 \pm 0.41$ & $13.22 \pm 0.30$ & $26.96 \pm 0.0 .67$ & $15.06 \pm 0.49$ & $11.03 \pm 0.24$ & $11.11 \pm 0.26$ & $64.16 \pm 1.51$ & 55.31 \\
\hline \multirow[t]{2}{*}{$\operatorname{High}(\geq 4)$} & \multirow[t]{2}{*}{2} & Knowledge & $8.00 \pm 0.00$ & $9.00 \pm 0.00$ & $17.00 \pm 0.00$ & $15.00 \pm 0.00$ & $22.00 \pm 0.00$ & $22.00 \pm 0.00$ & $76.00 \pm 0.00$ & 41.75 \\
\hline & & Adoption & $11.00 \pm 0.0$ & $10.00 \pm 0.00$ & $21.00 \pm 0.00$ & $10.00 \pm 0.00$ & $9.00 \pm 0.00$ & $9.00 \pm 0.00$ & $49.00 \pm 0.00$ & 42.24 \\
\hline
\end{tabular}

Table.7 Classification of respondents on the basis of social participation

\begin{tabular}{|c|c|c|c|c|c|c|c|c|c|c|}
\hline Category & $\begin{array}{l}\text { Percent of } \\
\text { Respondents }\end{array}$ & $\begin{array}{l}\text { Dependent } \\
\text { Variable }\end{array}$ & $\begin{array}{l}\text { General } \\
\text { Management } \\
\text { (Mean Score } \\
\pm \text { SE) }\end{array}$ & $\begin{array}{l}\text { Clean Milk } \\
\text { Production }\end{array}$ & $\begin{array}{l}\text { Total } \\
\text { Management }\end{array}$ & $\begin{array}{l}\text { Feeding } \\
\text { Practices }\end{array}$ & $\begin{array}{l}\text { Breeding } \\
\text { Practices }\end{array}$ & $\begin{array}{l}\text { Health- } \\
\text { Care } \\
\text { Practices }\end{array}$ & $\begin{array}{l}\text { Total score of } \\
\text { all Practices }\end{array}$ & $\begin{array}{l}\text { Scores } \\
\text { obtained in } \\
\text { Percentage }\end{array}$ \\
\hline \multirow{2}{*}{ Low $(\leq 0)$} & \multirow[t]{2}{*}{91} & Knowledge & $9.68 \pm 0.24$ & $11.14 \pm 0.38$ & $20.82 \pm 0.55$ & $19.97 \pm 0.53$ & $29.71 \pm 0.50$ & $22.64 \pm 0.42$ & $93.14 \pm 1.77$ & 51.17 \\
\hline & & Adoption & $13.45 \pm 0.37$ & $12.98 \pm 0.28$ & $26.43 \pm 0.61$ & $14.77 \pm 0.44$ & $10.74 \pm 0.21$ & $10.93 \pm 0.24$ & $62.87 \pm 1.35$ & 54.19 \\
\hline \multirow{2}{*}{$\begin{array}{l}\text { Medium } \\
(1-2)\end{array}$} & \multirow[t]{2}{*}{5} & Knowledge & $11.57 \pm 0.10$ & $13.14 \pm 1.12$ & $24.71 \pm 2.10$ & $21.86 \pm 2.47$ & $32.57 \pm 2.23$ & $26.43 \pm 0.92$ & $105.57 \pm 7.21$ & 58.00 \\
\hline & & Adoption & $16.57 \pm 1.25$ & $15.00 \pm 0.58$ & $31.57 \pm 1.77$ & $18.71 \pm 2.02$ & $13.14 \pm 0.63$ & $13.28 \pm 0.61$ & $76.71 \pm 4.86$ & 66.12 \\
\hline \multirow[t]{2}{*}{ High $(\geq 3)$} & \multirow[t]{2}{*}{4} & Knowledge & $10.00 \pm 0.00$ & $10.50 \pm 0.29$ & $20.50 \pm 0.29$ & $21.50 \pm 0.29$ & $37.00 \pm 0.58$ & $26.00 \pm 0.58$ & $105 \pm 1.73$ & 57.69 \\
\hline & & Adoption & $17.00 \pm 0.58$ & $16.00 \pm 0.58$ & $33.00 \pm 1.15$ & $20.50 \pm 0.29$ & $14.50 \pm 0.29$ & $13.50 \pm 0.29$ & $81.50 \pm 1.44$ & 70.25 \\
\hline
\end{tabular}


Table.8 Classification of respondents on the basis of extension contact

\begin{tabular}{|c|c|c|c|c|c|c|c|c|c|c|c|}
\hline Category & $\begin{array}{l}\text { Average } \\
\text { Score }\end{array}$ & $\begin{array}{l}\text { Percent of } \\
\text { Respondents }\end{array}$ & $\begin{array}{l}\text { Dependent } \\
\text { Variable }\end{array}$ & $\begin{array}{l}\text { General } \\
\text { Management } \\
\text { (Mean Score } \\
\pm \text { SE) }\end{array}$ & $\begin{array}{l}\text { Clean Milk } \\
\text { Production }\end{array}$ & $\begin{array}{l}\text { Total } \\
\text { Management }\end{array}$ & $\begin{array}{l}\text { Feeding } \\
\text { Practices }\end{array}$ & $\begin{array}{l}\text { Breeding } \\
\text { Practices }\end{array}$ & $\begin{array}{l}\text { Health- } \\
\text { Care } \\
\text { Practices }\end{array}$ & $\begin{array}{l}\text { Total score } \\
\text { of all } \\
\text { Practices }\end{array}$ & $\begin{array}{l}\text { Scores } \\
\text { obtained in } \\
\text { Percentage }\end{array}$ \\
\hline \multirow{2}{*}{$\begin{array}{l}\text { Low } \\
(\leq 3)\end{array}$} & \multirow[t]{2}{*}{$1-.5$} & \multirow[t]{2}{*}{16} & Knowledge & $8.21 \pm 0.44$ & $8.95 \pm 0.59$ & $17.16 \pm 1.02$ & $16.79 \pm 1.16$ & $27.31 \pm 1.27$ & $22.31 \pm 0.69$ & $83.58 \pm 3.90$ & 45.92 \\
\hline & & & Adoption & $13.00 \pm 0.77$ & $11.95 \pm 0.62$ & $24.95 \pm 1.33$ & $13.53 \pm 0.99$ & $9.47 \pm 0.51$ & $9.95 \pm 0.52$ & $57.89 \pm 3.22$ & 49.90 \\
\hline \multirow{2}{*}{$\begin{array}{l}\text { Medium } \\
(4-5)\end{array}$} & \multirow[t]{2}{*}{3.5} & \multirow[t]{2}{*}{80} & Knowledge & $9.94 \pm 0.24$ & $11.45 \pm 0.34$ & $21.39 \pm 0.56$ & $20.41 \pm 0.53$ & $30.28 \pm 0.52$ & $22.81 \pm 0.46$ & $94.89 \pm 1.79$ & 52.13 \\
\hline & & & Adoption & $13.61 \pm 0.40$ & $13.27 \pm 0.30$ & $26.88 \pm 0.65$ & $15.18 \pm 0.48$ & $11.10 \pm 0.22$ & $11.19 \pm 0.25$ & $64.36 \pm 1.43$ & 55.48 \\
\hline \multirow{2}{*}{$\begin{array}{l}\text { High } \\
(\geq 6)\end{array}$} & \multirow[t]{2}{*}{5} & \multirow[t]{2}{*}{4} & Knowledge & $12.67 \pm 0.92$ & $15.00 \pm 1.67$ & $27.67 \pm 2.59$ & $26.33 \pm 2.23$ & $36.33 \pm 0.21$ & $27.67 \pm 0.92$ & $118.00 \pm 5.84$ & 64.83 \\
\hline & & & Adoption & $18.33 \pm 1.17$ & $16.00 \pm 0.36$ & $34.33 \pm 1.28$ & $20.67 \pm 0.21$ & $14.33 \pm 0.21$ & $14.33 \pm 0.42$ & $83.67 \pm 1.65$ & 72.12 \\
\hline
\end{tabular}

Table.9 Classification of respondents on the basis of mass media exposure

\begin{tabular}{|c|c|c|c|c|c|c|c|c|c|c|c|}
\hline Category & $\begin{array}{l}\text { Average } \\
\text { Score }\end{array}$ & $\begin{array}{l}\text { Percent of } \\
\text { Respondents }\end{array}$ & $\begin{array}{l}\text { Dependent } \\
\text { Variable }\end{array}$ & $\begin{array}{l}\text { General } \\
\text { Management } \\
\text { (Mean Score } \\
\pm \text { SE) }\end{array}$ & $\begin{array}{l}\text { Clean Milk } \\
\text { Production }\end{array}$ & $\begin{array}{r}\text { Total } \\
\text { Management }\end{array}$ & $\begin{array}{l}\text { Feeding } \\
\text { Practices }\end{array}$ & $\begin{array}{l}\text { Breeding } \\
\text { Practices }\end{array}$ & $\begin{array}{l}\text { Health- Care } \\
\text { Practices }\end{array}$ & $\begin{array}{l}\text { Total score of } \\
\text { all Practices }\end{array}$ & $\begin{array}{l}\text { Scores } \\
\text { obtained in } \\
\text { Percentage }\end{array}$ \\
\hline \multirow{2}{*}{$\begin{array}{l}\text { Low } \\
(\leq 2)\end{array}$} & \multirow[t]{2}{*}{1} & \multirow[t]{2}{*}{28} & Knowledge & $9.82 \pm 0.43$ & $11.91 \pm 0.66$ & $21.73 \pm 1.04$ & $21.20 \pm 0.95$ & $30.56 \pm 1.02$ & $22.26 \pm 0.87$ & $95.76 \pm 3.21$ & 52.61 \\
\hline & & & Adoption & $13.35 \pm 0.77$ & $13.56 \pm 0.55$ & $26.91 \pm 1.20$ & $15.85 \pm 0.82$ & $11.32 \pm 0.39$ & $11.06 \pm 0.49$ & $65.14 \pm 2.51$ & 56.15 \\
\hline \multirow{2}{*}{$\begin{array}{l}\text { Medium } \\
(3-4)\end{array}$} & \multirow[t]{2}{*}{3} & \multirow[t]{2}{*}{53} & Knowledge & $9.17 \pm 0.27$ & $10.33 \pm 0.37$ & $19.50 \pm 0.63$ & $18.50 \pm 0.62$ & $28.76 \pm 0.61$ & $22.72 \pm 0.47$ & $89.48 \pm 2.08$ & 49.16 \\
\hline & & & Adoption & $13.47 \pm 0.41$ & $12.58 \pm 0.29$ & $26.05 \pm 0.67$ & $14.17 \pm 0.55$ & $10.37 \pm 0.26$ & $10.67 \pm 0.27$ & $61.26 \pm 1.65$ & 52.81 \\
\hline \multirow{2}{*}{$\begin{array}{l}\text { High } \\
(\geq 5)\end{array}$} & \multirow[t]{2}{*}{4.5} & \multirow[t]{2}{*}{19} & Knowledge & $11.59 \pm 0.49$ & $12.81 \pm 0.75$ & $24.41 \pm 1.22$ & $23.22 \pm 1.10$ & $33.36 \pm 0.88$ & $24.81 \pm 0.92$ & $105.81 \pm 3.86$ & 58.13 \\
\hline & & & Adoption & $15.18 \pm 0.92$ & $14.45 \pm 0.74$ & $29.64 \pm 1.59$ & $17.13 \pm 1.03$ & $12.36 \pm 0.48$ & $12.68 \pm 0.47$ & $71.81 \pm 3.32$ & 61.91 \\
\hline
\end{tabular}

Table.10 Classification of respondents on the basis of economic motivation

\begin{tabular}{|c|c|c|c|c|c|c|c|c|c|c|c|}
\hline Category & $\begin{array}{l}\text { Average } \\
\text { Score }\end{array}$ & $\begin{array}{l}\text { Percent of } \\
\text { Respondents }\end{array}$ & $\begin{array}{l}\text { Dependent } \\
\text { Variable }\end{array}$ & $\begin{array}{l}\text { General } \\
\text { Management } \\
\text { (Mean Score } \\
\pm \text { SE) } \\
\end{array}$ & $\begin{array}{l}\text { Clean Milk } \\
\text { Production }\end{array}$ & $\begin{array}{l}\text { Total } \\
\text { Management }\end{array}$ & $\begin{array}{l}\text { Feeding } \\
\text { Practices }\end{array}$ & $\begin{array}{l}\text { Breeding } \\
\text { Practices }\end{array}$ & $\begin{array}{l}\text { Health- } \\
\text { Care } \\
\text { Practices }\end{array}$ & $\begin{array}{l}\text { Total score } \\
\text { of all } \\
\text { Practices }\end{array}$ & $\begin{array}{l}\text { Scores } \\
\text { obtained in } \\
\text { Percentage }\end{array}$ \\
\hline \multirow{2}{*}{$\begin{array}{l}\text { Low } \\
(\leq 17)\end{array}$} & \multirow{2}{*}{17} & \multirow[t]{2}{*}{11} & Knowledge & $7.77 \pm 0.23$ & $8.92 \pm 0.37$ & $16.69 \pm 0.55$ & $18.08 \pm 0.77$ & $29.08 \pm 1.13$ & $22.77 \pm 0.77$ & $86.61 \pm 3.03$ & 47.58 \\
\hline & & & Adoption & $13.46 \pm 0.69$ & $12.69 \pm 0.54$ & $26.15 \pm 1.22$ & $15.69 \pm 0.82$ & $9.77 \pm 0.59$ & $10.38 \pm 0.49$ & $62.00 \pm 2.81$ & 53.45 \\
\hline \multirow{2}{*}{$\begin{array}{l}\text { Medium } \\
(18-22)\end{array}$} & \multirow[t]{2}{*}{20} & \multirow[t]{2}{*}{62} & Knowledge & $8.80 \pm 0.18$ & $10.10 \pm 0.31$ & $18.90 \pm 0.45$ & $18.21 \pm 0.45$ & $28.08 \pm 0.58$ & $21.04 \pm 0.46$ & $86.24 \pm 1.45$ & 47.38 \\
\hline & & & Adoption & $12.57 \pm 0.35$ & $12.18 \pm 0.32$ & $24.76 \pm 0.62$ & $13.56 \pm 0.50$ & $10.36 \pm 0.23$ & $10.30 \pm 0.25$ & $58.97 \pm 1.40$ & 50.84 \\
\hline \multirow{2}{*}{$\begin{array}{l}\text { High } \\
(\geq 23)\end{array}$} & \multirow[t]{2}{*}{23} & \multirow[t]{2}{*}{27} & Knowledge & $12.81 \pm 0.34$ & $14.69 \pm 0.58$ & $27.50 \pm 0.91$ & $25.37 \pm 1.00$ & $35.31 \pm 0.50$ & $27.19 \pm 0.50$ & $115.37 \pm 2.69$ & 63.39 \\
\hline & & & Adoption & $16.44 \pm 0.86$ & $15.62 \pm 0.43$ & $32.06 \pm 1.18$ & $18.69 \pm 0.82$ & $13.00 \pm 0.35$ & $13.37 \pm 0.39$ & $77.12 \pm 2.49$ & 66.48 \\
\hline
\end{tabular}


Int.J.Curr.Microbiol.App.Sci (2017) 6(12): 2758-2770

Table.11 Classification of respondents on the basis of risk orientation

\begin{tabular}{|c|c|c|c|c|c|c|c|c|c|c|c|}
\hline Category & $\begin{array}{l}\text { Average } \\
\text { Score }\end{array}$ & $\begin{array}{l}\text { Percent of } \\
\text { Respondents }\end{array}$ & $\begin{array}{l}\text { Dependent } \\
\text { Variable }\end{array}$ & $\begin{array}{l}\text { General } \\
\text { Management } \\
\text { (Mean Score } \\
\pm \mathrm{SE} \text { ) } \\
\end{array}$ & $\begin{array}{l}\text { Clean Milk } \\
\text { Production }\end{array}$ & $\begin{array}{l}\text { Total } \\
\text { Management }\end{array}$ & $\begin{array}{l}\text { Feeding } \\
\text { Practices }\end{array}$ & $\begin{array}{l}\text { Breeding } \\
\text { Practices }\end{array}$ & $\begin{array}{l}\text { Health- } \\
\text { Care } \\
\text { Practices }\end{array}$ & $\begin{array}{l}\text { Total score } \\
\text { of all } \\
\text { Practices }\end{array}$ & $\begin{array}{l}\text { Scores } \\
\text { obtained } \\
\text { in } \\
\text { Percentage }\end{array}$ \\
\hline \multirow{2}{*}{$\begin{array}{l}\text { Low } \\
(\leq 17)\end{array}$} & \multirow[t]{2}{*}{14.5} & \multirow[t]{2}{*}{12} & Knowledge & $8.00 \pm 0.21$ & $10.00 \pm 0.65$ & $18.00 \pm 0.74$ & $19.71 \pm 0.85$ & $31.57 \pm 1.43$ & $22.71 \pm 1.01$ & $92.00 \pm 3.36$ & 50.55 \\
\hline & & & Adoption & $14.43 \pm 0.75$ & $14.00 \pm 0.71$ & $28.43 \pm 1.43$ & $17.14 \pm 0.95$ & $10.57 \pm 0.57$ & $9.86 \pm 0.80$ & $66.00 \pm 2.74$ & 56.90 \\
\hline \multirow[t]{2}{*}{ Medium } & \multirow[t]{2}{*}{18.5} & \multirow[t]{2}{*}{69} & Knowledge & $9.35 \pm 0.24$ & $10.57 \pm 0.33$ & $19.92 \pm 0.54$ & $18.66 \pm 0.46$ & $28.51 \pm 0.54$ & $21.83 \pm 0.45$ & $88.92 \pm 1.67$ & 48.86 \\
\hline & & & Adoption & $12.75 \pm 0.35$ & $12.45 \pm 0.31$ & $25.20 \pm 0.62$ & $14.11 \pm 0.50$ & $10.81 \pm 0.26$ & $10.96 \pm 0.25$ & $61.09 \pm 1.52$ & 52.66 \\
\hline \multirow[t]{2}{*}{ High } & \multirow[t]{2}{*}{22.5} & \multirow[t]{2}{*}{19} & Knowledge & $12.54 \pm 0.48$ & $14.45 \pm 0.84$ & $27.00 \pm 1.31$ & $26.00 \pm 1.39$ & $35.45 \pm 0.69$ & $27.09 \pm 0.73$ & $115.54 \pm 3.83$ & 63.48 \\
\hline & & & Adoption & $16.91 \pm 1.10$ & $15.36 \pm 0.55$ & $32.27 \pm 1.53$ & $18.00 \pm 1.08$ & $12.09 \pm 0.45$ & $12.72 \pm 0.55$ & $75.09 \pm 3.35$ & 64.73 \\
\hline
\end{tabular}


The findings are in agreement of the findings of Fita et al., (2012), Hamdani (2008), Kapse (1976) and Rathod et al., (2014). It is conjectured here that the respondents engaged in diversified occupation activities could have higher mobility, diverse social interaction, different attitudinal sets, etc which in turn might have contributed to the differences observed.

\section{Herd Size}

For appropriate analysis respondents were classified into three categories based on their herd size i.e. low, medium and high (Table 6). Herd size was having positive and insignificant association with the knowledge whereas negative and insignificant association with adoption level (Table 1). As is evident from the table 6 the majority of respondents $(85 \%)$ were having medium herd size with 2 to 3 dairy animals, followed by low category (13\%) having less than 1 dairy animals.

The high herd size category was constituted by 2 percent of the respondents. The respondents with medium herd size obtained higher scores in knowledge while respondents with low herd size obtained higher scores in adoption compared to other two categories.

This is partialy in agreement with the findings of Hamdani (2008), Kunzru et al., (1987), Rathod et al., (2014), Sharma and Singh (2014), and Sohal and Tyagi (1978), who reported knowledge to be insignificantly associated with herd size.

This perhaps is a pointer to the growing presumption that the improved animal husbandry practices are not considered significantly beneficial by the farmers owing to the inappropriateness of the technologies to the present rearing system. However, this needs to be substantiated by appropriate studies.

\section{Social participation}

Data in table 1 shows that social participation was having positive but insignificant association with the knowledge level about animal husbandry practices. However, it was significantly positively associated with the adoption level. As is evident from the table 7 the majority of respondents $(91 \%)$ were having poor social participation. This is contrary to the popular belief that the extension flows from opinion leaders to peers, on which most of our extension systems are based. However, extent of social participation seems to have affected the adoption scores of respondents. It can be suggested here that the respondents with higher social participation scores in general have better resourcefulness, higher mobility, lesser change resistance and better cosmopoliteness. This in turn might have influenced the adoption scores of respondents with higher social participation. Jeelani et al., (2015), Mikkilineni (1976), Rathod et al., (2014) and Singh et al., (1989) reported social participation to be positively and significantly associated with animal husbandry practices in general.

Contrary to this, Sheoran (1987) and Chugh et al., (1996) observed insignificant association of social participation with adoption of animal husbandry practices. This probably is suggestive of the fact that there is significant variation in the pattern of social participation on the regional basis. Leadership patterns and extent of democratic values in the local socio cultural settings could have contributed to the differing reports.

\section{Extension contact}

Respondents were classified into three categories (i.e. low, medium and high) based on the extension contact scores obtained (table 8). Extension contact was having positive and significant association with both knowledge and adoption scores (Table 1). As is evident from the table 8 the majority of respondents $(80 \%)$ were having medium extension contact with mean value of 3.50 , followed by low extension contact group (16\%) and high contact group (4\%) with 1.5 and 5.0 mean values. Surprisingly the respondents with medium extension contact obtained higher scores in both knowledge and 
adoption compared to other two categories. The findings are in agreement with the findings of Hamdani (2008), Mikkilineni (1976), Prasad and Ponraj (2016) and Sharma and Singh (2014). The overall extension contact of the respondents in general was poor with a mean value of 4.22 . This could probably be used to hypothesize that with increase in the extension contact the knowledge and adoption level of respondents also increase as they became fully aware about benefits of improved animal husbandry practices. To begin with expansion of extension activities must be undertaken in a major way. The organization and performance of local extension systems could have been responsible for the varying reports. Studies to evaluate the efficacy of present extension system are strongly advocated.

\section{Mass media exposure}

Mass media exposure was having positive and highly significant association with knowledge level about improved animal husbandry practices. However, it was insignificantly positively associated with the adoption level (table 1).

Evidently, majority of respondents (53\%) were having medium mass media exposure, followed by low (28\%) and high (19\%) categories. Respondents with higher mass media exposure obtained significantly higher scores in both knowledge and adoption compared to other two categories. Similar findings were observed by Fita et al., (2012), Hamdani (2008), Jeelani et al., (2015). Mikkilineni (1976), Prasad and Ponraj (2016), Sharma and Singh (2014) and Sohal and Tyagi (1978). Thus it can be concluded here that mass media has pronounced effects on the knowledge and adoption of respondents. Thus it can be suggested that the mass media as an extension tool has a great potential. However, overall mass media exposure of the respondents was poor.

Appropriate steps to enhance the overall mass media exposure should form the first step prior to using mass media as an extension tool. This can perhaps be done by improving the quality and extent of mass media programmes.

\section{Economic motivation}

Economic motivation was having positive and highly significant association with both knowledge and adoption levels of the respondents (Table 1). As is evident from the table 10 the majority of respondents $(62 \%)$ were having medium economic motivation followed by high economic motivation group (27\%) and low motivation group (11\%). Surprisingly, respondents with high economic motivation obtained higher scores in both knowledge and adoption compared to other two categories. The differences, however, were not very wide. With the increase in the economic motivation of respondents there was little increase in the knowledge and adoption scores of respondents. Presumably it can be hypothesized that the respondents with high economic motivation perceive animal husbandry in general as a lucrative option. The findings are in agreement with the reports of Fita et al., (2012) and Tyagi and Sohal (1984) who observed positive and significant association of economic motivation with animal husbandry practices. However, Sawarkar et al., (2001) reported economic motivation to be negatively associated with the adoption. There perhaps is no uniform pattern of association between economic motivation and knowledge and adoption level of animal husbandry farmers. Perhaps the sustained growth of economy (especially service sector) provides suitable remunerative avenues to the people in general.

\section{Risk orientation}

Risk orientation was having positive and highly significant correlation with both knowledge and adoption levels of the respondents (table 1). As is evident from the table 11 the majority of respondents $(69 \%)$ were having medium level of risk orientation, followed by high (19\%) and low (12\%) categories. Surprisingly, respondents with high risk orientation obtained higher scores in both knowledge and adoption compared to 
other two categories. The findings are in agreement with the findings of Jeelani et al., (2015) and Rathod et al., (2014). Contrary to this Mahipal and Kherde (1989) reported risk orientation to be negatively and insignificantly related with animal husbandry practices. In any case, this is a pointer to the hypothesis that the livestock farmers willing to take risk were able to acquire their knowledge about improved animal husbandry practices making it an active effort. However, their adoption level remained unaffected. Probably the respondents were not convinced to translate the higher knowledge obtained into actual practice. It cannot however, be ruled out that the additional knowledge acquired was difficult to fit with the system of rearing. However, definite reasons behind this phenomenon remain covert in the absence of suitable literature and studies.

It can be concluded that young respondents have significantly higher knowledge than the respondents in old age. However, the age has no significant effect on the knowledge and adoption level regarding improved scientific animal husbandry practices. The statistical analysis of data revealed that knowledge and adoption level of respondents were significantly influenced by education, land holding, occupation, extension contact, economical motivation and risk orientation. Social participation and mass media exposure of the respondents varied with the varying levels of knowledge and adoption. Thus extension agencies need to intensively organize training, method demonstration, exhibition, group discussion, field tour etc. to increase adoption of improved animal husbandry practices at desirable level with emphasis on learning rather than teaching. Based on the findings the following recommendations are made for better knowledge and adoption of improved animal husbandry practices by dairy farmers.

Individual extension methods should be the focus on any future extension effort and emphasis should be laid on motivation as an inherent component.
Exposure visit, demonstration as well as providing on campus training programmes on improved animal husbandry practices to dairy farmers to aware and motivate them to adopt improved animal husbandry practices.

Information communication awareness on use of various Ict tools such as mobile internet, whatsapp, social media, web portals etc for easy access to good animal husbandry practices for rearing of dairy animals.

Organizing of more kissan mela, exhibitions.

\section{References}

Agricultural Labourers in Relation to Dairy Innovations. Indian Journal of Dairy Science. 42(4):707-711.

Bhalla and Hazzel 1998. Food Grains Demand in India to 2020: A preliminary Exercise. Economic and Political Weekly, 32(52): 150-154.

Birthal, P.S. and Parthasarty, R.P. 2002. Economic Contribution of Livestock Subsector in India. In Proceeding of ICAR- ICRISAT Collaboration Workshop held in January 2002.

Central Statistical Organization, CSO, Government of India, Livestock Census 2006-2007.

Chug, M. 1996. Exploratory study of dairy farm owned by ex-serviceman in Karnal District (Haryana). M.Sc., Thesis, National Dairy Research Institute, Karnal, Haryana (India).

Delgado, C., Rosegrant, M., Steinfeld, H., Ehui, S. and Courbois, C. 1999. Livestock to 2020: The Next Food Revolution. Food, Agriculture and Environment Discussion Paper 28 IFPRI /FAO /ILRI. IFPRI Washington DC

Fita, L., Trivedi, M. M., and Tassew, B. 2012. Adoption of Improved Dairy Husbandry Practices and its Relationship with the Socio-Economic Characteristics of Dairy Farmers In: Ada'a District of Oromia State, Ethiopia. Journal of Agricultural Extension and Rural Development, 
4(14):392-395.

Hamdani, S. A. 2008. Adoption Pattern of Improved Dairy Farming Practices in Jammu District. M.V.Sc. thesis. Sher-eKashmir University of Agricultural Sciences and technology of Jammu, J\&K. Jeelani, R., Khandi, S. A., Beig, M. Y., Bhadwal, M. S., and Kumar, P. 2015. Adoption of improved animal husbandry practices by Gujjars of Jammu and Kashmir. Indian Journal of Dairy Science, 68(3):287-291.

Kapse, S.S. 1976. Adoption of Selected Dairy Husbandry Practices of Cattle Owners in the ICDP area, Dhulia (Maharashtra). M.Sc. thesis, NDRI, Karnal, Haryana.

Kunzru, O. N., Sagar, R. L., Srivasthava, N. K. And Singh, P. 1987. Constraints in Adoption of haemorrhagic septicemia vaccine. Indian Journal of Extension Education, 23(3):74-76

Mahipal and Kherde, R.L. 1989. Correlates of adoption behaviour of dairy farmers. Maharashtra. Journal of Extension Education, 42(3): 434-439.

Mikkilineni, V. K. 1976. A study of adoption of selected dairy practices in upland and delta areas of Vijayawada Taluka (A.P). M.Sc. thesis. NDRI, Karnal, Haryana.

Paroda, R.S. and Kumar, P. 2000. Food production and demand in South Asia. Agricultural Economic Research Review. 13(1):1-24.

Prasad, N., and Ponraj, P. 2016. Study on adoption of scientific dairy farming. Advances in Animal and Veterinary Sciences, 4(2): 13-18.

Rao, B.S. 2002. A study of combined impact of dairy cooperatives and ICDP on Socioeconomic changes in milk shed area of
Bongir (A.P). M.Sc. Thesis (Unpub.), NDRI, Karnal.

Rathod, R. S., Singh, R. and Kachwaha, R. N. 2014. Adoption of recommended management practices and relationship between selected traits of the respondents. Indian Journal of Dairy Science, 62(4):327-334

Sawarkar, S.W., Borker, M.M., Upadhye, S.V. and Jadhao, S.B. 2001. Characteristics of dairy owners, their awareness, adoption and constraints in adoption of A.I. practices in Vidharba Region. Indian Journal of Dairy Science, 54(4): 194-202.

Sayeedi, A.G. 1983. A study of knowledge, attitude and adoption of dairy farmers towards dairy scientific activities in Jammu District (JandK State). Ph.D. Thesis Kurukshetra University, Kurukshetra.

Sharma, R. and Poleman, T. 1993. The new economics of the green revolution. Cornell University Press, Ithaca, USA.

Sharma, R. and Singh, T. 2014. The new economics of the green revolution. Cornell University Press, Ithaca, USA.

Sheoran, V. K. 1987. A Study of adoption of dairy innovations in relation to milk yield among beneficiaries of IRDP. Ph.D. thesis. NDRI, Karnal, Haryana.

Singh, S.P., Nirwal, R.S. and Singh, V.P. 1989. Adoption behaviour of farmers and Agricultural Labourers in Relation to Dairy Innovation.

Sohal, T.S. and Tyagi, K.C. 1978. Role of Knowledge in Adoption of Dairy Innovations. Indian Journal of Extension Education, 14(3): 16-25.

\section{How to cite this article:}

Sheikh Umair Minhaj, S.A. Khandi, Rayees Ahmed Bafanda, Bharat Bhushan and Farzana Choudhary. 2017. Correlation Analysis of Socio-Personal Profile of Dairy Farmers with Knowledge and Adoption of Improved Animal Husbandry Practices in Doda District of Jammu and Kashmir, India. Int.J.Curr.Microbiol.App.Sci. 6(12): 2758-2770.

doi: https://doi.org/10.20546/ijcmas.2017.612.320 